\title{
Sharp recanalization for long-segment superior vena cava occlusion in a hemodialysis patient with vascular resource exhaustion: a case report
}

\author{
Jing-Yi Wei ${ }^{1}$, Ling-Ling Wang ${ }^{1}$, Xue-Mei Zhang ${ }^{2}$, Tian-Lei Cui ${ }^{3}$ \\ ${ }^{1}$ West China School of Nursing/The Center of Gerontology and Geriatrics West China Hospital, Sichuan University , Chengdu, China; ${ }^{2}$ The \\ Center of Gerontology and Geriatrics, West China Hospital, Sichuan University, Chengdu, China; ${ }^{3}$ Division of Nephrology, Kidney Research \\ Institute, West China Hospital of Sichuan University, Chengdu, China \\ Correspondence to: Tian-Lei Cui. Division of Nephrology, Kidney Research Institute, West China Hospital of Sichuan University, \#37 Guoxue Alley, \\ Chengdu 610041, China. Email: tianleicui@163.com.
}

\begin{abstract}
Hemodialysis is the lifeline of end-stage renal patients, and the correct choice of vascular access is vital to patients with vascular resource exhaustion. A 57-year-old female was admitted to the hospital due to catheter dysfunction. Color-doppler ultrasound (CDU) showed that the patient's inferior vena cava (IVC), right brachiocephalic vein, and long segment of the superior vena cava (SVC) were occluded. During surgery, we found that the obstruction of the SVC extended from the opening of the azygous vein to the junction of the SVC and the right atrium and was $6.9 \mathrm{~cm}$ in length. Under fluoroscopic guidance, the original tunneled cuffed catheter (TCC) was pulled out, a 4-French sheath was implanted into the right, and a guidewire was inserted to locate the distal end of the SVC. Another puncture needle was inserted from the right brachiocephalic vein into the distal end of the SVC and the steel core of the Rosch-Uchida Transjugular Liver Access Set (RUPS-100) was then inserted through the puncture needle. After correcting the positive and lateral position and determining the way in which the SVC entered the right atrium from the distal end, a 0.035-inch hard guidewire was then inserted into the right atrium through the steel core and a 6-mm balloon was used to dilate the SVC. The end of the catheter was then implanted into the IVC. No surgical complications occurred and at the 30-month follow-up and time of writing, the catheter remained primarily patent. The use of the RUPS-100 for sharp recanalization of an occluded long segment of the SVC can increase the likelihood of patient survival, but the risks during this operation are still not negligible and require precise guidance.
\end{abstract}

Keywords: Case report; Rosch-Uchida Transjugular Liver Access Set (RUPS-100); sharp recanalization; superior vena cava (SVC); tunneled cuffed catheter (TCC)

Submitted Apr 22, 2021. Accepted for publication May 28, 2021.

doi: 10.21037/apm-21-1161

View this article at: https://dx.doi.org/10.21037/apm-21-1161

\section{Introduction}

In approximately $10 \%$ of patients with end-stage renal disease, a central vein catheter (CVC) is used for longterm dialysis to maintain life (1). Patients with a CVC may experience endothelial inflammation, fibrosis, and thrombus formation due to blood flow disturbances and mechanical stimulation caused by the insertion of a hemodialysis catheter, leading to intimal hyperplasia and occlusion of the SVC lumen. If the position of the catheter tip proximal to the right atrium, it may develop chronic occlusion of the SVC, or SVC syndrome due to the above reaction $(2,3)$. SVC occlusion can be treated via open surgical or endovascular repair. Although endovascular repair is associated with an increased need for secondary intervention, its decreased morbidity and equal effectiveness have made it a mainstay of treatment in recent years (4-6). 


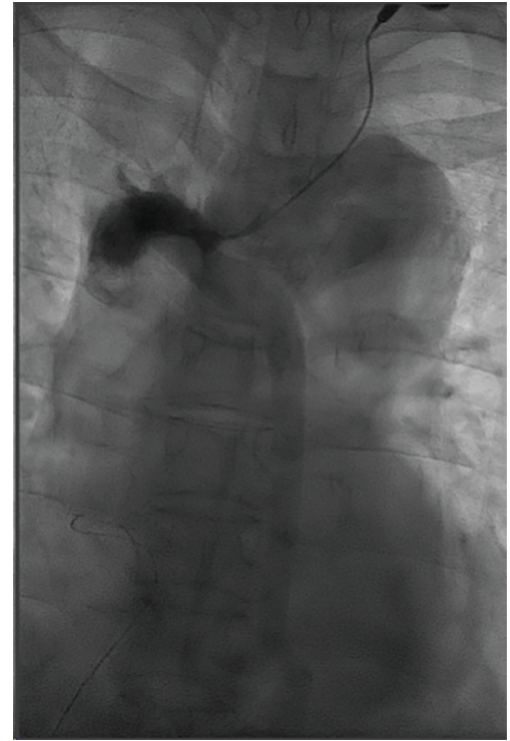

Figure 1 SVC occlusion identified by DSA. Venography via a left internal jugular vein approach. SVC, superior vena cava; DSA, digital subtraction angiography.

We report the case of a 57-year-old female suffering from vascular resource exhaustion and long segment SVC occlusion, and show the use of the RUPS-100 for sharp recanalization of the occluded SVC long segment increases the likelihood of patient survival. We present the following article in accordance with the CARE reporting checklist (available at https://dx.doi.org/10.21037/apm-21-1161).

\section{Case presentation}

A 57-year-old female patient with a history of multiple catheterizations and an internal fistula was admitted to the hospital because of TCC dysfunction in her left lower extremity. As infections and a thrombus due to long periods of CVC dialysis had nearly exhausted her current vascular access, the purpose of her admission was to seek long-term vascular access. The patient has been taking anticoagulants for a long time, and other family members have no kidneyrelated diseases.

While no positive signs were found on physical examination, chest computed tomography (CT) showed a low-density filling defect in the lower segment of the SVC and color-doppler ultrasound (CDU) showed that the patient's IVC, right brachiocephalic vein, and long segment of the SVC were occluded (Figure 1).

During the operation, the original TCC was pulled out, and a 4-French sheath was implanted into the right atrium. The reduction in atrial volume caused by the thrombus prevented the sheath from entering the SVC, so angiography was used to locate the vicinity of the junction between the right atrium and the SVC. Using a left internal jugular vein approach, this showed that the obstruction of the SVC, extending from the opening of the azygous vein to the junction of the SVC and the right atrium, was $6.9 \mathrm{~cm}$ in length, and the azygos vein was open. A guidewire was inserted to locate the distal end of the SVC, and another puncture needle was inserted from the right brachiocephalic vein into the distal end of the SVC. Steel core of the RUPS100 was then inserted through the puncture needle. After correcting the positive and lateral position and determining the way in which the SVC entered the right atrium from the distal end, a 0.035 -inch hard guidewire was inserted into the right atrium through the steel core (Figure 2) and a 6-mm balloon was used to dilate the SVC (Figure 3). The whole process was guided anteriorly and laterally by digital subtraction angiography (DSA). The end of the catheter was implanted into the IVC, and no surgical complications occurred.

After more than 2.5 years of follow-up, the catheter still functioned well.

Written informed consent was obtained from the patient for publication of this case report and any accompanying images. All procedures performed in studies involving human participants were in accordance with the ethical standards of the institutional and/or national research committee(s) and with the Helsinki Declaration (as revised in 2013).

\section{Discussion and conclusions}

The prerequisite for hemodialysis is to have a reliable vascular access, and the quality of the vascular access directly affects the patient's dialysis and quality of life. At present, there is no absolutely ideal type of vascular access. According to the recommendations of some international guidelines, autologous arteriovenous fistula (AVF) should be the first choice for vascular access. The establishment of AVF mainly includes three steps: selection of arteriovenous fistula position, selection of vascular anastomosis position and selection of AVF puncture timing. Common complications include vascular stenosis, acute thrombosis, venous hypertension, aneurysm, et al. Regular monitoring of vascular access and early intervention can reduce the occurrence of complications. For patients with upper arm 

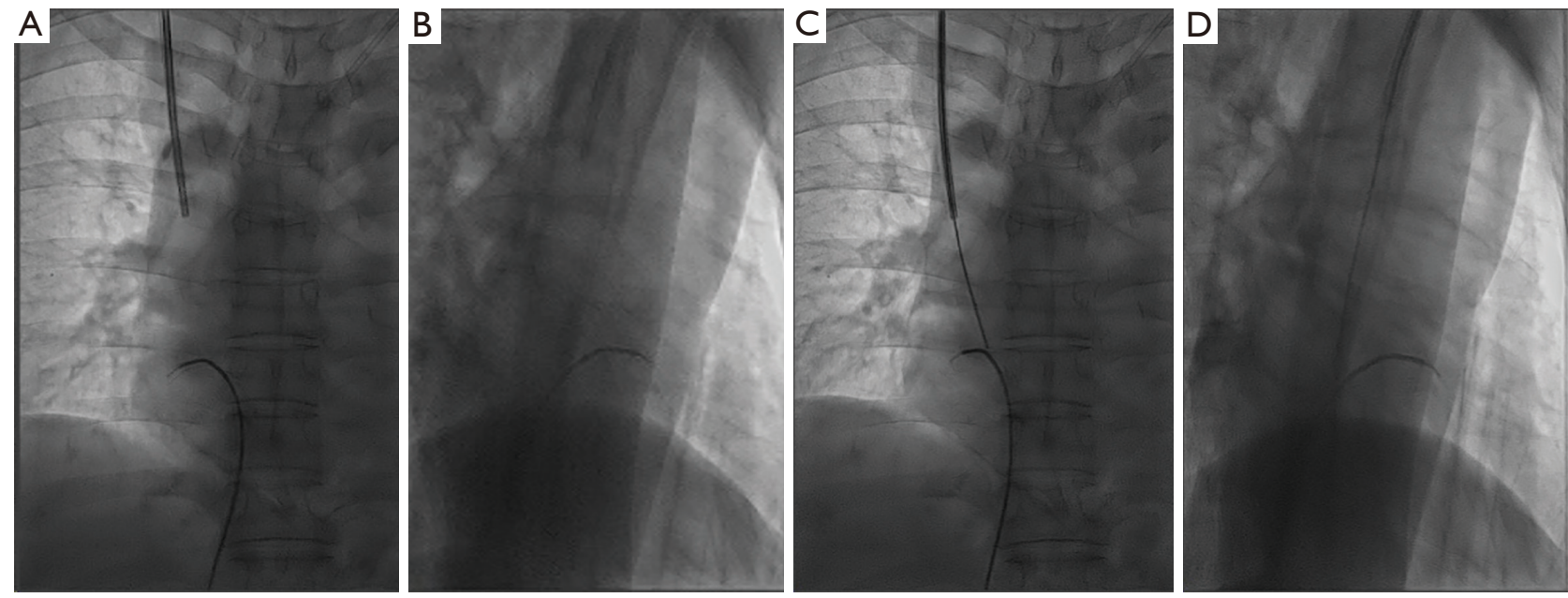

Figure 2 SVC occlusion sharp recanalization. (A) Anterior-posterior view before puncture; (B) side view before puncture; (C) anteriorposterior view after puncture; (D) side view after puncture. SVC, superior vena cava.
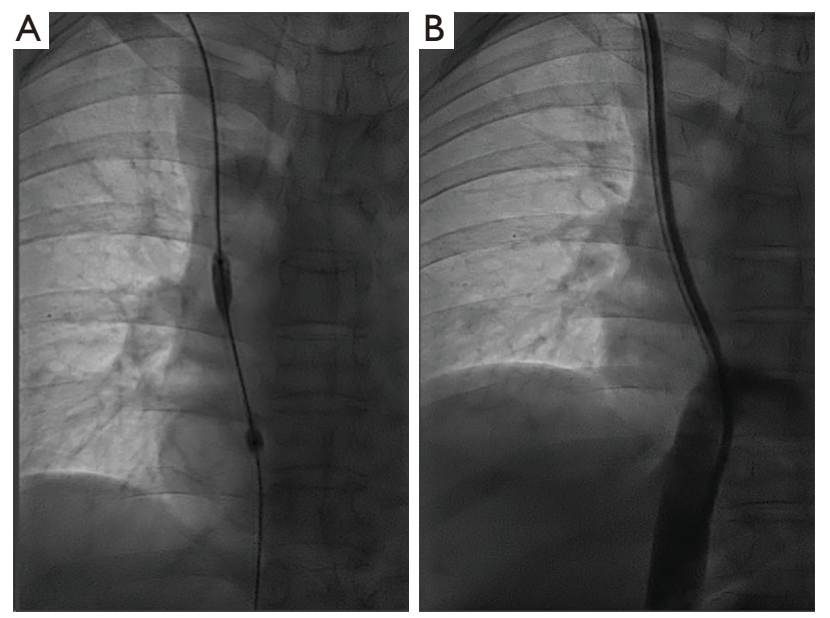

Figure 3 Balloon dilation and catheter insertion. (A) Occluded SVC dilated with balloons; (B) TCC implanted. SVC, superior vena cava; TCC, tunneled cuffed catheter.

vascular depletion, consider choosing arteriovenous graft (AVG) for the forearm or any type of vascular access in the upper arm. It is recommended that AVG of the forearm should be carried out first, which will help increase the diameter of the upper arm vein and increase the success rate of subsequent establishment of the upper arm AVF; for patients with upper extremity vascular depletion, consider choosing trunk AVG, lower extremity AVF or AVG.

When the autologous AVF cannot be established, AVG is the second choice, and CVC should be the last choice.
When patients need central venous catheterization, it is necessary to know whether the patients have heart failure, serious arrhythmia, shock, dyspnea and other critical conditions, whether they have a history of central venous catheterization and its puncture site, the number of catheterization, whether they have a history of infection, whether the operation process is smooth, and whether they have a tendency of severe bleeding, On the basis of comprehensive evaluation, the key to indwelling CVC is to select appropriate catheter length and straight position. For common complications such as puncture-related acute complications, thrombosis, infection, et al., it is necessary to adopt the correct puncture method during the catheterization process, ultrasonic positioning to guide the puncture catheterization, rational use of tube sealing liquid, strict aseptic technical operations, et al., to prevent.

The main points of the maintenance of hemodialysis vascular access are the prevention and treatment of longterm complications. The main long-term complications include catheter dysfunction, catheter infection and central vein stenosis/occlusion. The most common cause of catheter dysfunction is fibrin sheath and/or thrombosis. Good catheter placement technology and ideal catheter location are the most important to avoid this complication, Appropriate sealing technology is also an important guarantee to reduce the incidence of catheter dysfunction; Catheter infection requires timely diagnosis and treatment. Once a catheter-related infection is suspected, an etiological examination of the catheter lumen and peripheral blood 
should be performed immediately, and empiric antibiotics should be given through the vein or catheter, and the tube must be sealed with antibiotic solution. It is not recommended to remove the infected tunnel catheter without treatment to avoid loss of dialysis access; Central venous stenosis/occlusion has become a difficult problem in the complications of vascular access. When central venous stenosis/occlusion is suspected, the gold standard DSA should be used for diagnosis, and endovascular treatment should be performed at the same time. For patients with access loss, the access should be reconstructed in time or other transitional methods should be adopted to avoid central venous catheterization.

Even if the corresponding measures are adopted for prevention and treatment, some patients still inevitably have central venous catheter occlusion. Among them, SVC occlusion threatens the availability of vascular access for hemodialysis, and complicated bypass surgery is usually considered the last resort because of its high degree of comorbidity. While several endovascular techniques, including thrombolysis, percutaneous transluminal angioplasty, and stent placement, have been widely applied in the treatment of SVC occlusion in hemodialysis patients, the success rates and long-term patency rates of these technologies are variable (7). The use of a radiofrequency wire for the recanalization of the SVC has been reported in several case reports and series in the literature, but the risk with this technology is that the heated tip will allow the wire to easily advance extraluminally $(2,6)$. This can lead to two major complications; tracheal perforation and pericardial space entry (6). Transhepatic venipuncture and translumbar venipuncture are also treatment methods for refractory SVC obstruction, but have the disadvantages of poor tolerance and a high risk of infection. For patients refractory to conventional guidewire transversal, SVC sharp recanalization may be an option, and its application in SVC occlusion has been increasingly reported in recent years $(3,4)$.

In the present case, the junction of the SVC and right atrium was also blocked, resulting in a reduction in the volume of the right atrium. This makes it difficult to indwell the hepatic venous catheter, even if it is barely indwelled, and the therapeutic effect is not ideal. In addition, radiofrequency wire recanalization is more likely to burn into the inner wall, resulting in serious perforation and other complications. Zhao et al. reported on 16 hemodialysis patients who had undergone sharp recanalization of the brachiocephalic vein through the external jugular vein (EJV) suggested that when the internal jugular vein (IJV) is blocked, alternative vascular access such as the EJV can be considered. This method has good effectiveness $(100 \%)$ and safety $(87.5 \%)$. Compared with a brachial or femoral approach, the EJV holds the advantage of being adjacently located in a straight line to the brachiocephalic vein, which might enable using a straight sharp device during recanalization with better over-the-wire control (8). Therefore, transhepatic venipuncture and radiofrequency wire were not selected in this case, and a final attempt was made to use the RUPS-100 for sharp recanalization.

Although the operation was successful, there were still many risks, such as SVC laceration, hemopericardium, chest pain. The success of the operation and the good prognosis of the patient were likely due to the following factors: First, since the upper and lower central veins of the patient were almost completely blocked, transhepatic venipuncture would be used under normal circumstances, but we avoided this by choosing to retain the previously dysfunctional TCC as the guide. This may have increased patient tolerance and reduced patient suffering. Second, Ultrasound guided puncture catheters are relatively widely used in the puncture and recanalization of refractory indwelling catheters in elderly hemodialysis patients. It can avoid accidental injury to blood vessels, nerves and pleural apex during the operation. It has been reported in some cases $(4,9)$. The patient in this case had occlusion not only of the long segment of the SVC but also at the junction of the SVC and the right atrium, so accurate positioning was not possible. The risk of acute cardiac tamponade during surgery was also high, and to minimize this risk, we applied the forward and lateral real-time guidance of the DSA machine with double $\mathrm{C}$ arms and the plasticity of the end of the RUPS-100 steel core to make the process of sharp recanalization more controllable. At the same time, choosing a balloon that matches the diameter of the adjacent normal vein can reduce the risk of rupture and bleeding, as in this case the patient's right atrium was small, catheter tip insertion into the right atrium could not permit sufficient blood flow, so to maintain long-term hemodialysis function, a longer hemodialysis catheter was selected to be inserted into the IVC. The patient was followed up for more than 2.5 years, and the function of the catheter during reverse dialysis was good. This may be because the end of the TCC was placed in the lower cavity and the heparin administered with reverse dialysis could travel throughout the bloodstream, leading to a reduction in local fibrin and the rate of thrombosis in the IVC.

In conclusion, the use of the RUPS-100 for sharp 
recanalization of the occluded long segment of the SVC can increase the likelihood of patient survival, but the risks during this operation are still not negligible and require precise guidance.

\section{Acknowledgments}

The authors thank the patient for allowing us to publish this case report.

Funding: None.

\section{Footnote}

Reporting Checklist: The authors have completed the CARE reporting checklist. Available at https://dx.doi. org/10.21037/apm-21-1161

Conflicts of Interest: All authors have completed the ICMJE uniform disclosure form (available at https://dx.doi. org/10.21037/apm-21-1161). The authors have no conflicts of interest to declare.

Ethical Statement: The authors are accountable for all aspects of the work in ensuring that questions related to the accuracy or integrity of any part of the work are appropriately investigated and resolved. Written informed consent was obtained from the patient for publication of this case report and any accompanying images. A copy of the written consent is available for review by the Editor of this journal. All procedures performed in studies involving human participants were in accordance with the ethical standards of the institutional and/or national research committee(s) and with the Helsinki Declaration (as revised in 2013).

Open Access Statement: This is an Open Access article distributed in accordance with the Creative Commons Attribution-NonCommercial-NoDerivs 4.0 International License (CC BY-NC-ND 4.0), which permits the noncommercial replication and distribution of the article with the strict proviso that no changes or edits are made and the original work is properly cited (including links to both the formal publication through the relevant DOI and the license). See: https://creativecommons.org/licenses/by-nc-nd/4.0/.

\section{References}

1. Jin Q, Wang $\mathrm{Y}, \mathrm{Ye} \mathrm{C}$, et al. Consensus among experts on blood access used for hemodialyis in China (The 2nd edition). Chin J Blood Purif 2019;18:365-81.

2. Agarwal AK, Khabiri H, Haddad NJ. Complications of Vascular Access: Superior Vena Cava Syndrome. Am J Kidney Dis 2017;69:309-13.

3. Yang L, Yang L, Zhao Y, et al. The feasibility and safety of sharp recanalization for superior vena cava occlusion in hemodialysis patients: A retrospective cohort study. Hemodial Int 2020;24:52-60.

4. Shwaiki O, Khoncarly S, Buchino JJ, et al. Sharp Recanalization of a Chronically Occluded Superior Vena Cava in a Patient with Multiple Prior Peripherally Inserted Central Catheters. J Assoc Vasc Access 2019;24:21-6.

5. Majdalany BS, Monfore N, Khaja MS, et al. Radiofrequency Wire Recanalization of Chronically Occluded Venous Stents: A Retrospective, Single-Center Experience in 15 Patients. Cardiovasc Intervent Radiol 2019;42:130-6.

6. Keller EJ, Gupta SA, Bondarev S, et al. SingleCenter Retrospective Review of Radiofrequency Wire Recanalization of Refractory Central Venous Oclusions. J Vasc Interv Radiol 2018;29:1571-7.

7. Kundu S. Review of central venous disease in hemodialysis patients. J Vasc Interv Radiol 2010;21:963-8.

8. Zhao Y, Lin F, Yang L, et al. Sharp recanalization of the brachiocephalic vein occlusion through the external jugular vein in hemodialysis patients. Ann Transl Med 2020;8:640.

9. Tonak J, Fetscher S, Barkhausen J, et al. Endovascular recanalization of a port catheter-associated superior vena cava syndrome. J Vasc Access 2015;16:434-6.

(English Language Editor: B. Draper)
Cite this article as: Wei JY, Wang LL, Zhang XM, Cui TL. Sharp recanalization for long-segment superior vena cava occlusion in a hemodialysis patient with vascular resource exhaustion: a case report. Ann Palliat Med 2021;10(7):8518-8522. doi: 10.21037/apm-21-1161 